\title{
The New World IoT Is Bringing Forth
}

\author{
Shuichi FUKUDA ${ }^{1}$ \\ Keio University, Systems Design and Management Research Institute
}

\begin{abstract}
The current discussion about IoT is basically an advanced RFID framework along the line of traditional engineering, i.e., machines and humans work separately. Humans are outside of the machine system and give instructions. Machines respond to them. But true IoT removes the wall between humans and machines and they start fo work together as members on the same team. Fhus, IoT calls fof communication between humans and machines. Machines do not wait for human instructions and they understand what humans expect from them and act accordingly. This paper describes how true IoT will change our world and create the new world, World 2.0, where humans and machines form a team to respond to the frequently and widely changing environments and situations.
\end{abstract}

Keywords. IoT, Man-Machine Mixed Teamworking, Communication-rich, Integration of Material and Life Science, World 2.0

\section{Introduction}

This paper points out true IoT will create the new world. This "World 2.0" is a communication-rich society, and humans and machines work together as members on the same team. In short, life is infused into machines in the World 2.0.

How different the World 2.0 is from the current world is described, and what challenges we have and how we may possibly overcome them are discussed.

\section{Engineering Yesterday, Today and Tomorrow}

Figure 1 shows engineering yesterday and today. Yesterday, the world is small and closed with boundaries. But today the world is open and boundaries disappear. And it is expanding rapidly as shown in Figure 2.

When the world is small and bounded, we could solve the problems with mathematical rational approaches easily. But as the world expands, it became increasingly difficult.

Engineers realized the same approach can be applied as the one we use to identify the name of a river. If we look at the river itself, we cannot identify its name, because water is changing continuously. But if we look around, we can find mountains or forests, which do not change. So, we can identify its name, based on these feature points (Figure 3).

\footnotetext{
${ }^{1}$ Corresponding Author, Eail: shufukuda@gmail.com.
} 


\section{Traditional Engineering Individual Products}

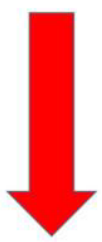

IoT Connected Engineering

Team Products

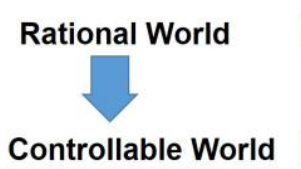

Increasing

Degree of Freedom

Explicit Knowledge

\section{Things Team}

Tacit Knowledge

Figure 1. Engineering yesterday, today and tomorrow.

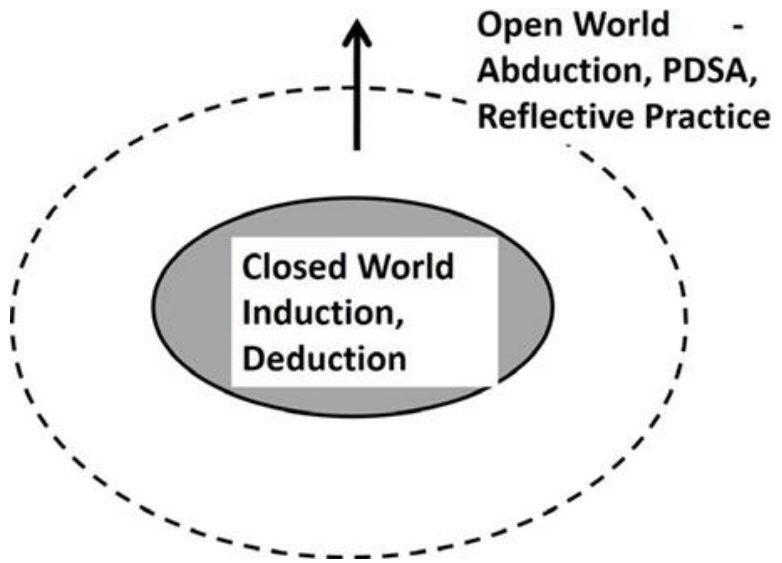

Figure 2. Closed World to Open World.

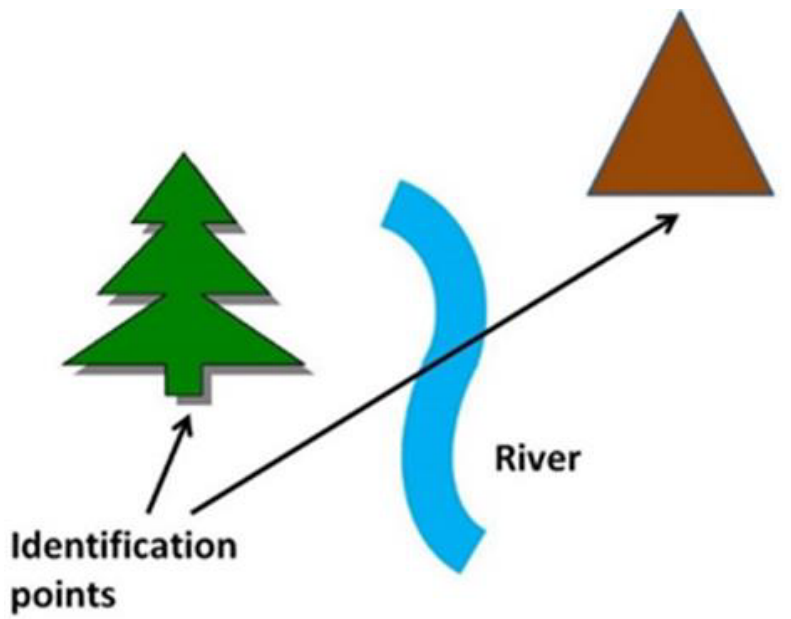

Figure 3. How can we identify the name of a river?

Thus, engineers succeeded in expanding the rational world and established the controllable world (Figure 4). 


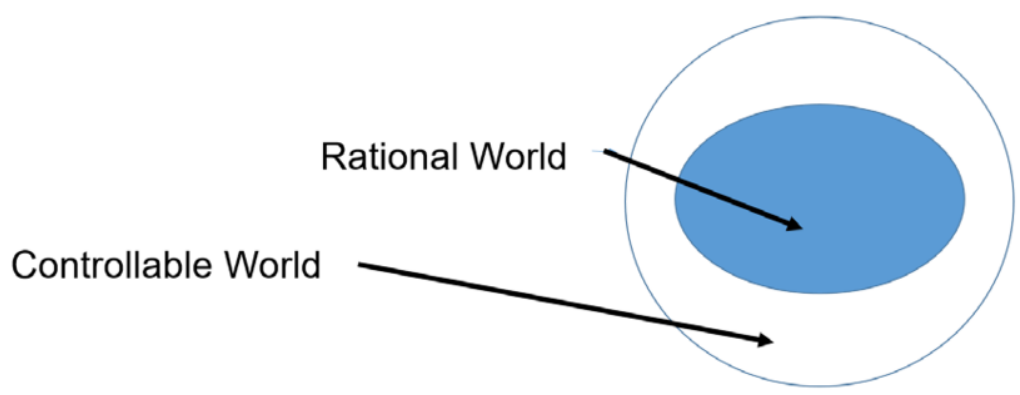

Figure 4. Rational World to Controllable World.

But the speed of the world expansion increases exponentially and our world shifted from verbal or explict to nonverbal or tacit. Bicyle riding is often taken up as an example of tacit knowledge [1], but in that case, the outer world can be described explicitly. Therefore, swimming is a better example.

So, although we expanded rational world by avoiding the difficulty of describing the behavior of water, we are, so to speak, brought back to the problem of how we describe it.

\section{Human Motion Control}

We have been discussing how we expanded rational world, focusing our attention on the outer world. Here, let us turn our eyes to human motions. The difficulty of describing the behavior of water is its almost infinitely large number of degrees of freedom. So, we turned our eyes from water to mountains, forest, etc. which do not change, and succeeded in reducing the number of degrees of freedom.

In the case of human motions, the same issue comes up. Figure 5 shows the famous cyclogram of Nikolai Bernstein. As it shows, human motion trajectories vary very widely. Thus, the degrees of freedom was a big problem. Bernstein indicated coordination plays an important role in human motion control [2].

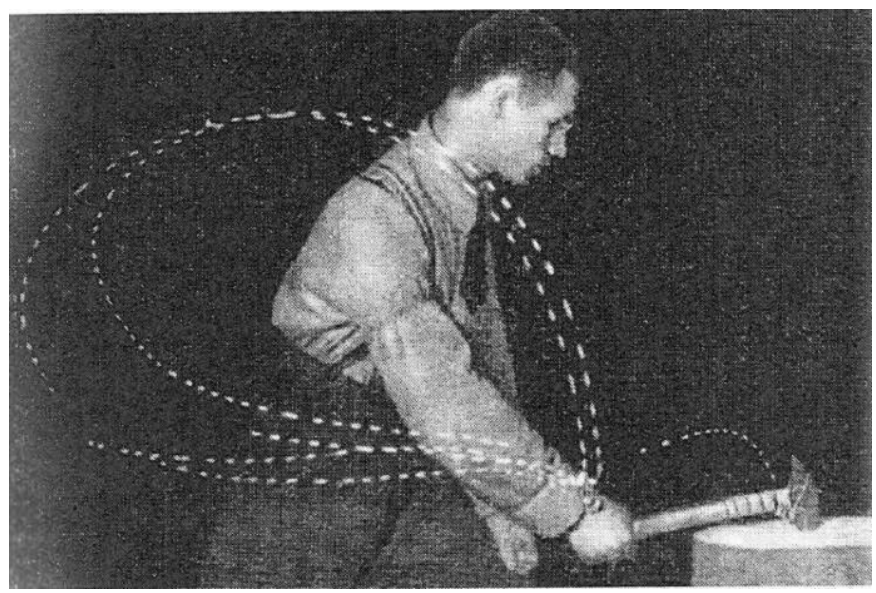

Figure 5. Cyclogram of hammering. 
Although he did not point out, we note that when the hammer comes close to the target, human motion trajectory is fixed and it does not vary from time to time. At this stage, the number of degrees of freedom is reduced to the minimum. Our rational approaches were developed, focusing our attention only on this stage.

Yesterday, the situations did not change appreciably, so we could focus our attention only on this last stage and apply rational approaches. But situational changes are no more changes of yesterday.

\section{Changes Are Changing}

We should take note that changes are changing.

Yesterday, there were changes, too. But they were smooth. So, we could differentiate them mathematically. Thus, we could predict the future.

Today, changes not only take place frequently and extensively, but they change sharply. So, we cannot differentiate them. Therefore, we cannot predict the future.

Whether we can predict the future or not is the big difference between changes of yesterday and today (Figure 6).

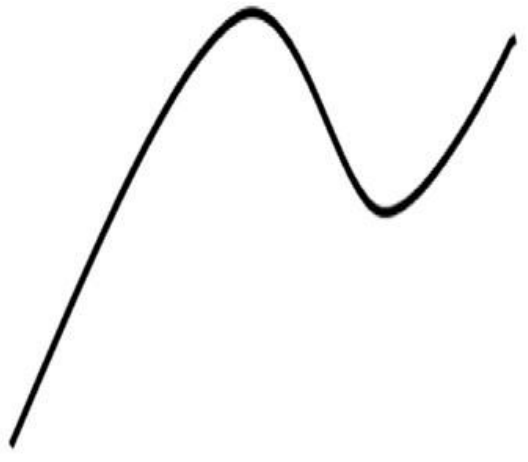

Yesterday

\section{Smooth Change Differentiable Predictable}

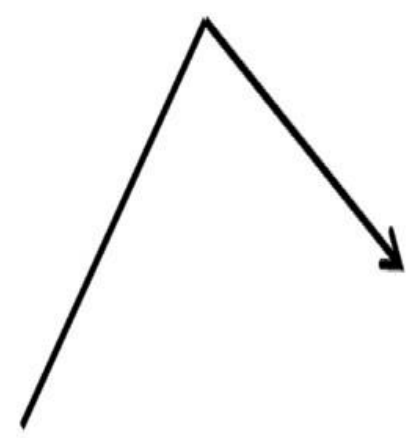

Today

\section{Sharp Change Not Differentiable Not Predictable}

Figure 6. Changes of yesterday and today.

\section{Outside of the System to In the System}

As we could predict the future, we could operate machines from outside. But now we cannot predict the future, we need to know what is happening right now on the spot. So, we need to work in the system (Figure 7). 

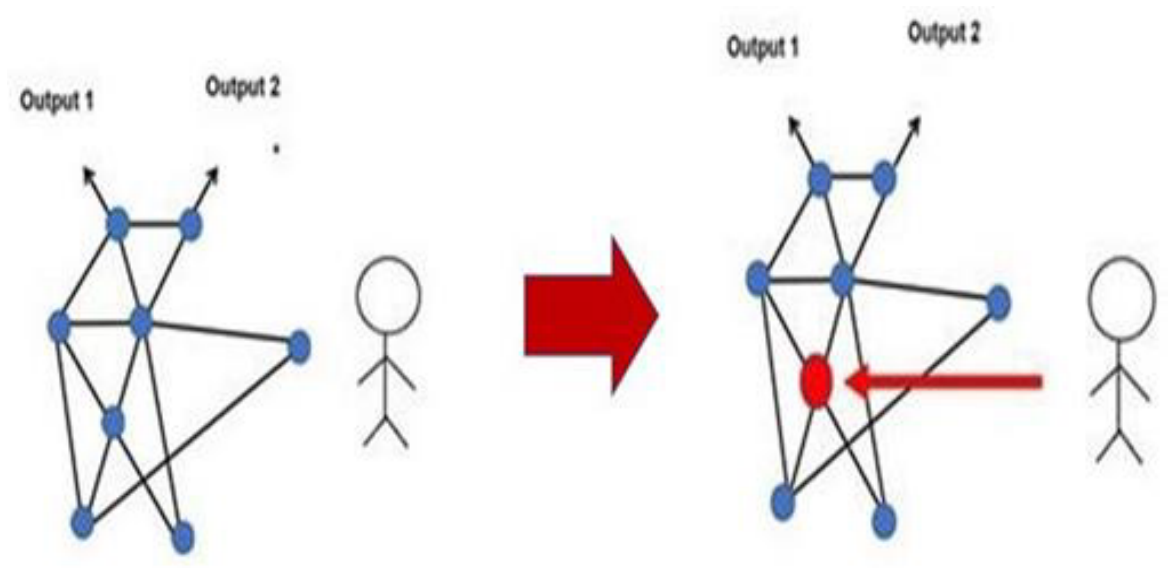

Figure 7. Outside of the System to In the System.

But until very recently, our way of working in the system has been the same as we did yesterday. We instructed machines and machines responded. The way of message passing did not change. We only needed to know how the situatons change. That is why we started to work in the system.

Let us take soccer for example. Yesterday, the situations did not change appreciably in the game. So, the formation did not change during the game and each player was expected to play his best in his assigned position.

But today, situations change frequently. So, we cannot give instructions from outside of the pitch. Only players know what is happening now and how they should adapt to it. Thus, midfielders are now playing-managers. Managers are now playing on the pitch.

We must remember, however, that every player needs to understand what the playing-manager expects from him and prepare beforehand. Oherwise, the strategy does not work. In other words, communication is strongly called for in the current game

Now messages are transferred not in signal passing or in conversation style, but in communication style. This is what Knute Rockne, American football coach and player, said " 11 Best to Best 11". Soccer is shifting from 11 Best to Best 11. Players on the team understand and share the strategies by communication.

\section{Conversation to Communication}

To adapt to the changing situations adequately, we need to introduce communication as soccer demonstrates. Thus, IoT (Internet of Things) emerged [3]. Internet is a tool for communication. Until its emergence, humans and machines changed messages, but in a conversation or message passing way. Humans give instructions and machines responded. But IoT changed it to communication. Machines now understand what humans expect from them and they operate without explicit instructions. And they can give suggestions or advice to humans for them to perform better to achieve what humans have on their minds. 


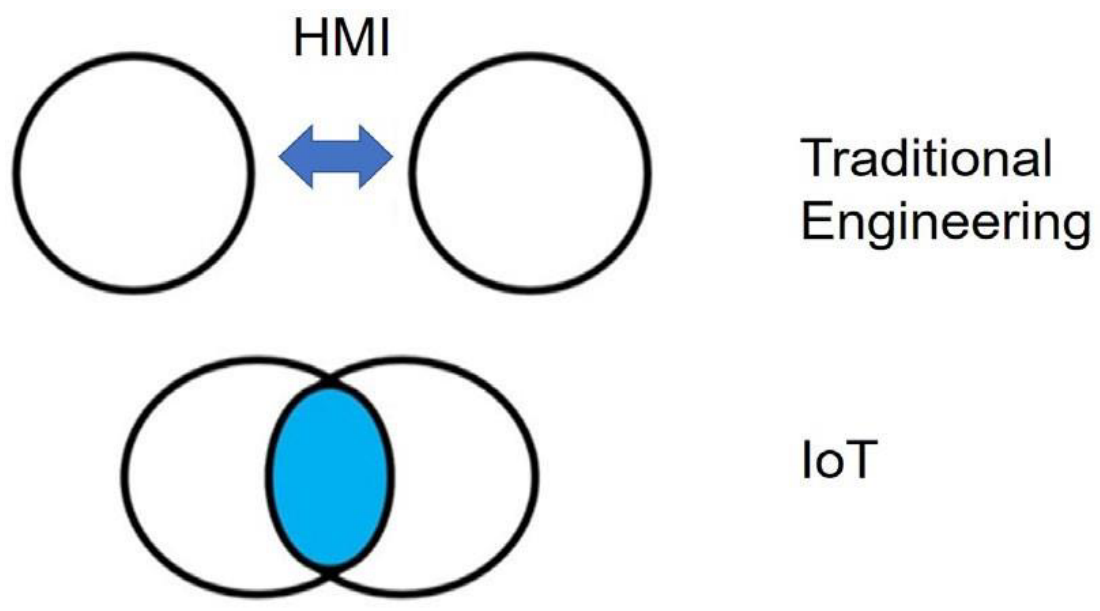

Machine

Human

Figure 8. Traditional Engineering and IoT.

\section{Humans and Machines Work Together on the Same Team in the IoT Connected Society}

As IoT is communication-driven, humans need to work together with machines on the same team. This is very much revolutionary. We have never experienced working togeher with machines on the same team. Indeed, we worked together with machines, but it was as physical materials. But IoT infused life into machines.

Until now, material science and life science are separate. But from now on, we need to integrate them.

\section{How IoT Will Change Our Life}

IoT is often discussed in the traditional RFID framework. But this is totally wrong. Why IoT is revolutionary is it removed the barrier between Material and Life Science. Machines are no more nonliving things. Now they are living.

We used machines based on our knowledge. But knowledge is structured experience of the past. What we need now is not knowledge, but wisdom. We need to perceive and recognize the situations correctly and make adequate actions to adapt to them. As the situations are unpredictable, we need wisdom. Experience of the past does not work anymore.

Sharing economy is attracting wide attention these days. This is another wisdom. We expected particular roles or functions from products, but if we look at them from different angles, they offer us many different roles or functions.

IoT changes experience from the thing of the past to the thing of the future. Now, we are creating experience. We can experience many different kinds of life. So IoT will 
bring us the world of sharing life. We are no more stuck with such constraints as regions, etc. We can create new experiences with life-infused machines and products.

\section{Forming an Adaptive Network}

We are now forming a team with machines. In other words, we need to form an adaptive network. But unlike sports, this team does not have any rules about the number of players or about the game.

Traditionally, the number of team members are fixed and there were rules, not only in sports, but in other organizations as well. So, the current AI works quite well.

But IoT teams have no such constraints. Its goal is how we can build up a team to adapt to the frequently and extensively changing situations. Thus, forming a most adaptable network is the goal, and besides we do not have any rules. Therefore, we do not care about how we get there. All's well that ends well. IoT is a pragmatic engineering. This is indeed a big challenge and there are many issues to overcome.

But if look at it from another perspective, it is life science. Living things make tremendous efforts to adapt to the changing situations in order to expand their community or to expand the world of their species. It is evolution. Evolution is nothing other than sustainability and adaptability.

And IoT will increase the importance of decision making. Economists point out that the quinary sector of the economy is decision making as shown in Table 1.

Table 1. Five sectors of the economy.

\begin{tabular}{ll}
\hline Sector & Activities \\
\hline 5 Quinary Sector & Decision Making \\
\hline 4 Quaternary Sector & Knowledge and ICT Industry \\
\hline 3 Tertiary Sector & Service Industry \\
\hline 2 Secondary Sector & Transforms raw materials into Products - Manufacturing, etc. \\
\hline 1 Primary Sector & Extracts raw materials from nature - Agriculture, Fishing, etc. \\
\hline
\end{tabular}

But economists discuss decision making in the current society framework. We need to be aware that the IoT Connected Society is completely different.

Decision making there is something more like human motion control in water. How we can coordiate our body parts and control our motions. We need to make decisions by trial and error. Learning from failures play an important role there.

\section{IoT and Human Motion Control: How Can We Do Better Next Time?}

As discussed in Chapter 2, coordination is called for in human motion control. But we do not have any supporting tools. There are many measuring equipment, but they only show us the successful motion control. But in reality, we achieve such a motion control by trial and error. We need a tool to help us to know how much we are improving. This is associated with learning from failures.

In fact, how we balance our body is not clear yet. It is associated with deep sensation or proprioception and this still remains unsolved in physiology. But apart from such scientific discussion, we can swim. We can balance our body. That is 
because we know how much better we are doing this time. We understand instinctively how much we are improving.

If we have a tool that provides us with a quantitative measure how much we are improving, then that will help us a great deal to learn how we can coordinate our motion. This holds true with IoT. IoT introduces human-machine mixed teamworking, so it needs coordination of system elements or parts.

P. C. Mahalanobis proposed Mahalanobis Distance (MD). MD is a measure of the distance between a point $\mathrm{P}$ and a distribution $\mathrm{D}$. It is a multi-dimensional generalization of the idea how many standard deviations away point $\mathrm{P}$ is from the mean $\mathrm{D}$ [4]. Therefore, it is unitless Genichi Taguchi noted that patterns can be identified by MD [5],[6]. His system only discussed static identification of patterns. Shuichi Fukuda expanded his idea to dynamic motion control [7]. As human body is composed of musculoskeletal system, we can easily represent muscles and skeletons as system parts and if we measure by MD how these parts change their patterns, we know how much we are improving or how much we are deteriorating. We can learn from failures.

Figure 9 and Figure 10 show the basic idea of Mahalanobis Taguchi System. We define Unit Space, which is a typical pattern. And we measure MD. If the MD decreases, then we are improving and if it increases, we are deteriorating. And if the MD falls within the threshold, our coordination is successful. We do not care how successful it is. If MD is within the threshold, then we are happy, because we succeeded in coordinating our body parts and in controlling our motions.
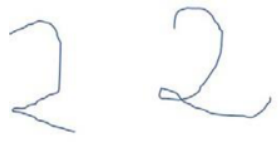

Unit Space

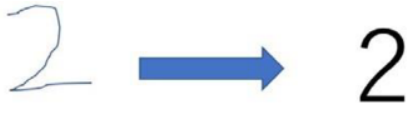

\section{Average Unit Space}

Figure 9. Defining Unit Space.

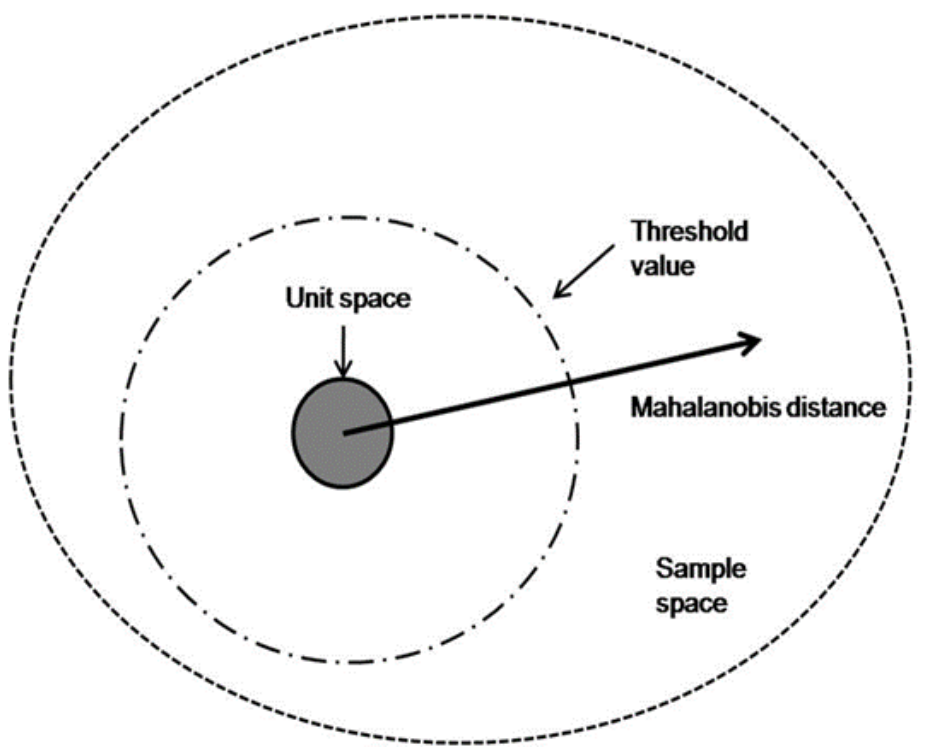

Figure 10. Mahalanobis Distance. 


\section{Summary}

This paper describes how the world will change with the true IoT. IoT is not another advanced RFID, It integrates nonliving things and living things and create a new world "The World 2.0", which we have never experienced.

In the World 2.0, experience is not the thing of the past, but it will be the thing of the future. We create experience with life-infused machines and products.

\section{References}

[1] M. Polanyni, The Tacit Dimension, University of Chicago Press, 2009.

[2] N.A. Bernstein, The Co-ordination and Regulation of Movements, Pergamon Press, Oxford, 1967.

[3] K. Ashton, "That 'Internet of Things' Thing”, RFID Journal, 22, June, 2009.

[4] https//en.wikipedia.org/wiki/Mahalanobis_distance.

[5] G. Taguchi, J. Chowdhury and Y. Wu, The Mahalanobis Taguchi System, McGraw-Hill Professional, New York, 2000.

[6] G. Taguchi and R. Jugulum, The Mahalanobis-Taguchi Strategy: A Pattern Technology System, Wiley, Hoboken, 2002.

[7] S. Fukuda, Self Engineering: Learning from failures, Sprinter, London, 2019. 